\title{
Infections After Liver Transplantation: A Retrospective, Single-center Study
}

\author{
M. Antunes ${ }^{a}$, A. Teixeira ${ }^{a}$, P. Fortuna ${ }^{a}$, B. Moya ${ }^{a}$, A. Martins ${ }^{a}$, L. Bagulho ${ }^{a}$, J.P. Pereira ${ }^{b}$, L. Bento ${ }^{a}$, \\ R. Perdigoto ${ }^{b}$, E. Barroso ${ }^{b}$, and P. Marcelino ${ }^{a, *}$

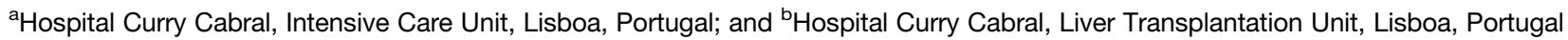

\begin{abstract}
Objective. To access the incidence of infectious problems after liver transplantation (LT).

Design. A retrospective, single-center study.

Materials and Methods. Patients undergoing LT from January 2008 to December 2011 were considered. Exclusion criterion was death occurring in the first 48 hours after LT. We determined the site of infection and the bacterial isolates and collected and compared recipient's variables, graft variables, surgical data, post-LT clinical data.

Results. Of the 492 patients who underwent LT and the 463 considered for this study, 190 (Group 1, 41\%) developed at least 1 infection, with 298 infections detected. Of these, 189 microorganisms were isolated, $81(51 \%)$ gram-positive bacteria (most frequently Staphylococcus spp). Biliary infections were more frequent (mean time of $160.4 \pm 167.7$ days after LT); from 3 months after LT, gram-negative bacteria were observed (57\%). Patients with infections after LT presented lower aminotransferase levels, but higher requirements in blood transfusions, intraoperative vasopressors, hemodialysis, and hospital stay. Operative and cold ischemia times were similar.

Conclusion. We found a $41 \%$ incidence of all infections in a 2-year follow-up after LT. Gram-positive bacteria were more frequent isolated; however, negative bacteria were commonly isolated later. Clinical data after LT were more relevant for the development of infections. Donors' variables should be considered in future analyses.
\end{abstract}

$\mathbf{I}^{\mathrm{N}}$ NFECTIOUS diseases are a common problem after solid organ transplantation, especially after liver transplant (LT) [1]. Infections cause significant morbidity and mortality, which affect graft survival [2]. The described rate is variable, with most of them being bacterial infections $[3,4]$.

Many factors are taken into account. These include donor variables (active infection, prolonged intensive care unit [ICU] stay, and acute kidney injury); graft variables (its quality and presence of steatosis); recipient data variables (such as poor nutritional status, clinical characteristics [model for end stage liver disease, MELD, and ChildTurcotte-Pugh $\{$ CTP $\}$ scores]) [5-8]; and intraoperative data (volume of transfused blood, and ischemia-reperfusion injury [IRI]) [9]. All these patients start immunosuppression early, so more infectious complications can develop after LT.

In recent years, many improvements have taken place regarding organ allocation (especially after the introduction of the MELD score) [10], surgical technique, antimicrobial and antifungal prophylaxis, and immunosuppression [11]. Nonetheless, the frequency of infectious events after LT did not decrease significantly [12]. Regional differences may exist; thus, the infectious events can vary from center to center as well as between countries. Data from various centers, then, help to obtain a clearer picture of this particular problem in these patients.

Our liver transplant center is a leading center in the country. In this study, the rate of all infections after LT is described, in a 2-year follow-up.

*Address correspondence to Paulo Marcelino, Hospital Curry Cabral, Intensive Care Unit, Rua da Benificiência, 8; 1069-166 Lisboa, Portugal. E-mail: par.marcelino@gmail.com

$0041-1345 / 15$ http://dx.doi.org/10.1016/j.transproceed.2015.03.009 


\section{MATERIALS AND METHODS}

All patients who underwent LT from January 2008 to December 2011 were considered for enrollment. As well as patients who died intraoperatively, patients who died in the first 48 hours after LT were excluded.

Patients who undergo LT in our center receive antimicrobial prophylaxis with ceftazidime $1 \mathrm{~g}$, and amoxicillin and clavulanic acid $1.2 \mathrm{~g}$, both intravenously and 3 times a day; oral nystatin every 6 hours; and, for females, vaginal clotrimazole once a day. In selected patients (defined by the presence of at least 1 of the following conditions: urgent LT due to acute liver failure or acute-on-chronic liver failure; serum creatinine $>2 \mathrm{mg} / \mathrm{dL}$ prior to LT or hemodialysis preceding LT; early acute kidney injury [AKI] after LT with need for renal replacement technique; retransplantation; early postLT need for surgical reintervention [mainly due to vascular complications and bleeding]; or more than $40 \mathrm{U}$ of transfused cellular blood components), prophylaxis with liposomal amphotericin B $(\mathrm{L}-\mathrm{AmB})$ was given as an intravenous administration of $100 \mathrm{mg}$ daily for 14 days. The usual immunosuppressive therapy used in our center consists of prednisolone, in a dose of $3 \mathrm{mg} / \mathrm{kg}$ i.v. in the first days, decreasing $20-30 \mathrm{mg} /$ day until reaching the maintenance dose of $20 \mathrm{mg} /$ day; mycophenolate mophetil, $500 \mathrm{mg}$ b.i.d. (according to platelet and white blood cell count); calcineurin inhibitors CNI (cyclosporine A, $8 \mathrm{mg} / \mathrm{kg} /$ day or tacrolimus, $0.1 \mathrm{mg} / \mathrm{kg} / \mathrm{day}$ ), to attain a target serum level of 350-400 $\mathrm{ng} / \mathrm{mL}$ for CYA and 8-12 ng/ $\mathrm{mL}$ for TCR. Patients with renal impairment previous to LT received basiliximab $4 \mathrm{mg}$ in the first day and $4 \mathrm{mg}$ in the fourth postoperative day; in these patients, $\mathrm{CNI}$ is introduced after 7 days. The hepatologist prescribes all the immunosuppressors.

The data collected were recipient's age; gender; operative time; cold ischemia time; pre-LT MELD and CTP scores; development of acute kidney injury (AKI) after LT (using the AKI classification); hemodynamic instability during surgery (defined as the continuous infusion of vasopressor for more than 5 minutes); maximum alanine aminotransferase (ALT) and aspartate aminotransferase (AST) levels after LT; number of RBC units transfused during surgery; ICU and hospital stay; and type of LT (deceased-donor LT or sequential LT). All infections were considered during the study period, determined by the presence of a documented site of infection and/or isolated infectious agent.

In these cases, several samples of body fluids were collected for microbiological analysis on suspicion of infection. For study purposes, they were divided as follows: biliary tract infections; respiratory infections; urinary tract infections; bloodstream infections (BSI); surgical wound infections; undetermined infections (febrile patients, in whom antibiotics were used, but without a proven site of infection or microbial isolates); opportunistic infections (caused by typically opportunistic agents, such as cytomegalovirus or Pneumocystis jiroveci); and fungal infections. These infections also were analyzed, to determine the mean days of detection after LT, the most common pathogens found (except for undetermined infections), and the antibiotics used, by order of frequency. A separate analysis was performed for patients presenting biliary tract infections and respiratory tract infections.

Deceased-donor liver grafts were harvested from brain-dead, heart-beating donors (the majority from other Portuguese institutions), preserved in Celsior solution and implanted in the recipient using the piggyback technique. In domino liver transplantation (DLT), the native hepatectomy in familial amyloidotic polyneuropathy (FAP) patients and the implantation of the deceased-donor graft were also done in the standard piggyback fashion, with retrohepatic vena cava preservation and without venovenous bypass. FAP livers also were flushed with and maintained in the Celsior solution. As FAP liver grafts were harvested without the vena cava, the hepatic venous outflow of the domino grafts was reconstructed on the back table, using a vein graft from the deceased donor as previously described [13]. Following the reconstruction of the hepatic venous outflow, the portal vein, hepatic artery, and bile duct were anastomosed.

All patients undergoing LT must sign an informed consent. The present protocol for data collection was reviewed and approved by the institution's ethics board.

Continuous variables are expressed as an average and standard deviation. Categorical variables are presented as categories with percentages. For comparative analysis, parametric (Student $t$ test, for numeric variables, after confirmation of the normal distribution of data by the Kolmogorov-Smirnov test) and nonparametric tests $\left(\chi^{2}\right.$ test or Fisher exact test, for categorical variables) were used. To establish dependence between variables, logistic regression analysis was performed (backward-LR), using variables that were significant on univariate analysis, and a goodness-of-fit test (Hosmer-Lemeshow) was used to assess the fit of the logistic regression model. A $P$ value $<.05$ was considered statistically significant. Statistical analysis was performed using SPSS 19.0 (Statistical Package for Social Sciences Inc., Chicago, Ill., United States).

\section{RESULTS}

In the study period, 492 patients underwent LT. Of these, 29 died either during surgery or in the first 48 hours after LT and were excluded. For study purposes, a total of 463 patients were considered. From these, 190 (41\%) developed at least 1 infection, and 63 developed a second or more infection during the follow-up period. Overall, 298 infections and 189 identifications of infectious agents were registered.

In Table 1 the comparative characteristics of the patients are shown, divided by those who did not present any infectious disease and those who did. We observed that in patients who developed infections the aminotransferases level was lower; nonetheless, the overall hospital stay was higher. Group 1 patients showed a higher mean AKI level, not reaching statistical significance, but renal replacement techniques were more often used. Deaths were higher in Group 1 ( 29 vs $18, P=.001$ ), although the overall survival was not significantly different, by the end of the study period. In Group 1, 21 deaths were attributable to infectious causes, all due to sepsis and multiorgan failure. Of these, 14 presented undetermined site of infection and/or no bacterial isolates, 1 presented biliary infection, 1 intra-abdominal infection, 2 BSI, and 1 surgical wound infection. Two other patients presented irreversible respiratory failure due to P.s jiroveci infection. There were similar graft losses ( 3 in Group 1, 4 in Group 2) and retransplantation (2 in each group). The mean time from LT to death was $190 \pm 377$ days in group 1 (10 occurred in the first 3 months), and $164 \pm 209$ in Group 2 ( 7 occurred in the first 3 months).

In Table 2, the incidence of infections is shown, divided by location, time after LT, and isolated agents. Overall, 189 microorganisms were isolated, separated by the Gram strain, and major species of microorganisms. We note that 
Table 1. Patients Characteristics, Separated by the Presence of Infectious Diseases

\begin{tabular}{lcc}
\hline \multicolumn{1}{c}{ Parameter } & Patients With Infection $(\mathrm{n}=190)$ & Patients Without Infection $(\mathrm{n}=273)$ \\
\hline Age (years, mean and SD) & $47.3 \pm 12.6$ & $46.5 \pm 12.6$ \\
Main pathologies ( $\mathrm{n}$ and \%) & & $16(5.5 \%)$ \\
$\quad$ Acute liver failure & $6(3.4 \%)$ & $215(74.5 \%)$ \\
Compensated cirrhosis & $126(72.5 \%)$ & $58(20 \%)$ \\
FAP & $42(24.1 \%)$ & $161(55.7 \%)$ \\
Deceased-donor recipients ( $\mathrm{n}$ and \%) & $110(63.2 \%)$ & $13.4 \pm 4$ \\
MELD score (mean and SD) & $14.4 \pm 4.2$ & $6.5 \pm 2.2$ \\
CTP score (mean and SD) & $6.6 \pm 2.3$ & $321 \pm 60$ \\
Operative time (minutes, mean and SD) & $325 \pm 63$ & $371 \pm 63$ \\
Cold ischemia time (minutes, mean and SD) & $387 \pm 68$ & $1652 \pm 1838$ \\
Maximum ALT (IU, mean and SD) & $1287 \pm 1150$ & $2593 \pm 3572$ \\
Maximum AST (IU, mean and SD) & $1948 \pm 1973$ & $75(25.9 \%)$ \\
Intraoperative hemodynamic instability (n and \%) & $72(41.3 \%)$ & $3.8 \pm 4.2$ \\
RBC (units, mean and SD) & $4.9 \pm 4.5$ & $0.84 \pm 1.1$ \\
Mean AKI score (mean and SD) & $1.1 \pm 1$ & $8(2.7 \%)$ \\
Hemodialysis ( $n$ and \%) & $7(4 \%)$ & $5.9 \pm 7.2$ \\
ICU stay (days, mean and SD) & $5.25 \pm 4.1$ & $19.3 \pm 9.4$ \\
Hospital stay (days, mean and SD) & $23.7 \pm 12$. & $18(6.5 \%)$ \\
Deceased (at 2 years) & $29(15.2 \%)$ & $1023 \pm 426$ \\
Mean survival after LT (at 31st December 2012; & $913 \pm 584$ & .025 \\
days, mean and SD) & & .001 \\
\hline
\end{tabular}

Abbreviations: SD, standard deviation; FAP, familial amyloidotic polyneuropathy; MELD, Model for End-stage Liver Disease; CTP, Child-Turcotte-Pugh; ALT, alanine aminotransferase; AST, aspartate aminotransferase; IU, international units; RBC, red blood cell; AKI, acute kidney injury; ICU, intensive care unit.

Staphylococcus aureus spp were more frequently and earlier isolated. As shown in Table 3, of the bacterial agents, grampositive agents were more frequent in the first 90 days after LT, whereas gram-negative agents were more frequent thereafter.

Regarding patients with biliary tract infection, it was not found to be a significant difference, apart from a longer hospital stay $(28.3 \pm 13$ vs $20.5 \pm 10.5 ; P=.011)$ and lower AST level $(1740 \pm 1534$ vs $2401 \pm 3181 ; P=.05)$. The group of patients with respiratory tract infection were older $(51.4 \pm 11.1$ vs $46.5 \pm 12.6 ; P=.026)$ and stayed longer in the hospital $(28.6 \pm 9.2$ vs $20.9 \pm 11 ; P=.023)$.

By logistic regression analysis, no individual parameter was found to be independently related to the considered dependent variables: occurrence of infection, occurrence of biliary tract infection, or occurrence of respiratory tract infection.

\section{DISCUSSION}

This study, performed in a single LT center, revealed a $41 \%$ prevalence of infection in a 2-year follow-up. The most frequent were biliary tract infections, with a mean time of occurrence of $160.4 \pm 167.7$ days after transplant. The most frequently isolated were gram-positive bacteria, although gram-negative bacteria prevailed in urinary tract infections and were more frequently isolated after the first 90 days after LT. These data are in general accordance with that described in the literature, although other authors describe a higher incidence of infections. Piselli et al. [14] described a rate of $56.3 \%$ and Souza et al. [15] reported a rate of $55.3 \%$, whereas Vera et al. [16] reported an incidence of 55.3\%. In another period, after 1 year post LT, Aberjet et al. [17] described an incidence of $31 \%$ of infections. Note that these studies were conducted in different countries and continents and represented different periods after LT. So, local conditions are critical to the pattern of infectious diseases in this group of patients, and this knowledge is valuable in treating them at a particular LT center. Grampositive bacteria are also described as the main bacterial isolates fond in these patients, particularly Staphylococcus spp. Also, some authors noticed a shift toward higher rate of gram-negative isolates. However, we found that these were more frequent later after LT.

A relatively high number of patients presented infectious problems without an identifiable point of infection or bacterial isolate. By the time of occurrence, they were very close to BSI, so we may surmise that most of these cases are BSI, although not detected by microbiological analyses [18,19].

Aminotransferase levels after LT depend on graft quality (one of the donor's related variables), harvesting, and cold ischemia time, as well as on intraoperative data, such as operative time, blood transfusion requirement (hemorrhage), and need for vasopressors (the hallmark of hemodynamic instability). These were described by Howard et al. as preservation injury [20]. We observed that aminotransferase were higher in the group that did not develop any infection, and only the need for vasopressors was higher in Group 1. This was true even for biliary tract infection. We analyzed these cases separately, assuming that there could be a link between preservation injuries, ischemia-associated 
Table 2. Infections Distributed by Infection Site and Time After LT

\begin{tabular}{|c|c|c|c|}
\hline Infection Site & & & \\
\hline Infectious Agent & After LT & After LT & Total \\
\hline Biliary tract & 28 & 26 & 54 \\
\hline Gram negative & 15 & 15 & 30 \\
\hline Acinetobacter baumanii & 4 & 3 & 7 \\
\hline Escherichia coli & 2 & 3 & 5 \\
\hline Pseudomonas aeruginosa & 1 & 3 & 4 \\
\hline Klebsiella pneumoniae & 2 & 1 & 3 \\
\hline Enterobacter cloacae & 2 & 1 & 3 \\
\hline Other & 3 & 4 & 7 \\
\hline Gram positive & 15 & 11 & 26 \\
\hline Enterococcus faecium & 9 & 6 & 15 \\
\hline Methicillin-resistant Staph. aureus & 3 & 0 & 3 \\
\hline Enterococcus faecalis & 2 & 1 & 3 \\
\hline Methicillin-sensitive Staph. aureus & 0 & 3 & 3 \\
\hline Other & 1 & 1 & 2 \\
\hline Intra-abdominal & 6 & 2 & 8 \\
\hline Gram negative & 3 & 1 & 4 \\
\hline Pseudomonas aeruginosa & 2 & 0 & 2 \\
\hline Enterobacter cloacae & 1 & 0 & 1 \\
\hline Citrobacter freudii & 0 & 1 & 1 \\
\hline Gram positive & 3 & 1 & 4 \\
\hline Enterococcus faecium & 2 & 1 & 3 \\
\hline Clostridium difficile & 1 & 0 & 1 \\
\hline Operative wound & 13 & 0 & 13 \\
\hline Gram negative & 2 & 0 & 2 \\
\hline Serratia marcencens & 1 & 0 & 1 \\
\hline Proteus mirabilis & 1 & 0 & 1 \\
\hline Gram positive & 11 & 0 & 11 \\
\hline Methicillin-resistant Staph. aureus & 8 & 0 & 8 \\
\hline Methicillin-sensitive Staph. aureus & 3 & 0 & 3 \\
\hline Urinary tract & 21 & 18 & 39 \\
\hline Gram negative & 15 & 17 & 32 \\
\hline Escherichia coli & 9 & 12 & 21 \\
\hline Klebsiella pneumoniae & 5 & 4 & 9 \\
\hline Pseudomonas aeruginosa & 1 & 0 & 1 \\
\hline Enterobacter cloacae & 0 & 1 & 1 \\
\hline Gram positive & 6 & 1 & 7 \\
\hline Enterococcus faecium & 4 & 0 & 4 \\
\hline Enterococcus faecalis & 2 & 1 & 3 \\
\hline Respiratory & 8 & 9 & 17 \\
\hline H1N1 & 0 & 1 & 1 \\
\hline Gram negative & 3 & 3 & 6 \\
\hline Acinetobacter baumanii & 1 & 1 & 2 \\
\hline Klebsiella pneumoniae & 1 & 0 & 1 \\
\hline Streptococcus pyogenes & 1 & 0 & 1 \\
\hline Haemophilus influenzae & 0 & 1 & 1 \\
\hline Pseudomonas aeruginosa & 0 & 1 & 1 \\
\hline Gram positive & 5 & 5 & 10 \\
\hline Methicillin-resistant Staph. aureus & 4 & 4 & 8 \\
\hline Vancomycin-resistant enterococci & 1 & 0 & 1 \\
\hline Methicillin-sensitive Staph. aureus & 0 & 1 & 1 \\
\hline Bloodstream & 17 & 4 & 21 \\
\hline Gram negative & 3 & 1 & 4 \\
\hline Acinetobacter baumanii & 2 & 0 & 2 \\
\hline Pseudomonas aeruginosa & 1 & 0 & 1 \\
\hline Stenotrophomonas maltophilia & 0 & 1 & 1 \\
\hline
\end{tabular}

Table 2. (continued)

\begin{tabular}{lccr}
\hline Infection Site & $\begin{array}{c}\text { First } \\
\text { 90 Days } \\
\text { After LT }\end{array}$ & $\begin{array}{c}\text { From } \\
\text { 90 Days } \\
\text { After LT }\end{array}$ & Total \\
\hline Gram positive & 14 & 3 & 17 \\
Methicillin-resistant Staph. aureus & 9 & 3 & 12 \\
Enterococcus faecalis & 2 & 0 & 2 \\
Staphylococcus epidermidis & 2 & 0 & 2 \\
Enterococcus faecium & 1 & 0 & 1 \\
Opportunistic & 4 & 10 & 14 \\
CMV & 4 & 3 & 7 \\
Pneumocistis jirovecii & 0 & 4 & 4 \\
Varicella & 0 & 3 & 3 \\
Other & 0 & 7 & 17 \\
Gram positive & 0 & 7 & 17 \\
Methicillin-resistant Staph. aureus & 0 & 4 & 4 \\
Methicillin-sensitive Staph. aureus & 0 & 3 & 3 \\
Fungal Infection & 5 & 0 & 5 \\
Candida & 5 & 0 & 5 \\
TOTAL & 103 & 76 & 189 \\
\hline
\end{tabular}

biliary disease after LT, and infection in this location. Even baseline patients' characteristics do not explain this fact, since patients with acute liver failure and FAP patients were similar in each group. One can conclude that graft-related factors and probably donor-related factors can contribute to the development of infections after LT. We could not find any comparative data in the literature.

Recipient characteristics also did not influence the occurrence of infections. The clinical data (MELD and CTP score) and all baseline characteristics were similar in both groups. Some data in the literature describe higher incidence of infections in patients with higher MELD scores; perhaps in these patients, often with acute or acuteon-chronic disease, bacteriological surveillance is higher and they could receive more antimicrobial prophylaxis or more aggressive therapy; frequently they undergo LT with ongoing antibiotic treatment. There are conflicting reports on the altered infection patterns after introduction of MELD score. This study was not designed to answer this question, but the main issue is that patient characteristics did not interfere with the occurrence of infections.

Regarding surgical data, the only variables significantly different in the group that developed infection were the need for vasopressor support, a surrogate for intraoperative hemodynamic instability, and RBC requirements. Nonetheless, these events can contribute to a worsened clinical

Table 3. Gram Stain Isolates by Time From LT

\begin{tabular}{|c|c|c|c|c|c|c|}
\hline & \multicolumn{2}{|c|}{$\begin{array}{l}\text { First } 90 \text { Days } \\
\text { After LT }\end{array}$} & \multicolumn{2}{|c|}{$\begin{array}{l}\text { From } 90 \text { Days } \\
\text { After LT }\end{array}$} & \multicolumn{2}{|c|}{ TOTAL } \\
\hline & $n$ & $\%$ & $n$ & $\%$ & $n$ & $\%$ \\
\hline Gram & 53 & $51 \%$ & 28 & $37 \%$ & 81 & $43 \%$ \\
\hline Gram negative & 41 & $40 \%$ & 37 & $49 \%$ & 78 & $41 \%$ \\
\hline
\end{tabular}


course early after LT and are not necessarily related to graft quality or preclinical conditions. We could not find a similar description in the literature. Nonetheless, this hemodynamic instability can particularly affect the post-LT clinical course, including graft function. A higher RBC requirement also is described in the literature as a risk factor for infections after LT.

Regarding the clinical course after LT, patients with infections had a similar incidence of AKI, although they required renal replacement techniques more often. The need for dialysis is a known risk factor for the development of infections after LT, both bacterial and fungal. Although the AKI score was similar, it was slightly higher in patients who developed infection; more patients in this group showed more severe renal injury, requiring increased use of dialysis. The greater requirement of vasopressor support during surgery in this group may also be linked to the greater requirements in dialysis, thus affecting the clinical course after LT. Also, these patients had longer hospital stays but not in the ICU. This condition may reflect the above-mentioned facts, the time of occurrence of infection after LT (after ICU discharge), and dialysis requirement but can also be the consequence of more infections detected and resulting in delayed hospital discharge.

The occurrence of opportunistic infections is in accordance with that described in the literature [21]. Fungal infections appeared earlier, but other opportunistic infections appeared later $[22,23]$.

\section{Study Limitations}

We could not access the donor's data, apart from the donation from FAP patients, a factor that did not influence the occurrence of infections. These data could be critical to understand or add new risk factors to those already known. In future studies, this fact may be a subject for separate analysis.

A large group of unknown isolates was found. Although cultures are requested frequently, and most often bacterial infection is suspected and the infection site is detected, the specific agent is not always isolated. Other diagnostic tools for infections, using molecular diagnosis, could be useful in these cases.

Most studies distinguish early and late infections after LT. We decided to provide full data, but also presented the mean time of occurrence of all infectious sites, so we assume that it would be easy to identify them. We also describe that gram-negative bacteria are more common in later infections, thus occurring later after LT.

\section{CONCLUSION}

In this retrospective study, we found a $41 \%$ prevalence of infection in a 2-year follow-up. The most frequent were undetermined infections, and most deaths were due to sepsis with multiorgan failure. Biliary tract infections were frequent, with a mean time of occurrence of $160.4 \pm 167.7$ days after transplant. More prevalent were Gram positive bacteria, although Gram negative bacteria were more frequently isolated later after LT. The aminotransferase levels were higher in patients without infection, whereas dialysis requirement and hospital stay were higher in patients who presented with infections. Donor variables could not be assessed and deserve a separate analysis in future studies.

\section{REFERENCES}

[1] Fishman JA, Rubin RH. Infections in organ-transplant recipients. N Engl J Med 1998;338:1741.

[2] Cockbain AJ, Goldsmith PJ, Gouda M, Attia M, Pollard SG, Lodge JP, et al. The impact of postoperative infection on long-term outcomes in liver transplantation. Transplant Proc 2010;42:4181-3.

[3] Colonna JO, Winston DJ, Brill JE. Infectious complications in liver transplantations. Arch Surg 1998;123:360-4.

[4] Paya C, Hermans PE, Larson-Keller JJ, Ilstrup DM, Krom RA, Rettke S, et al. Risk factors for cytomegalovirus and severe bacterial infection following liver transplantation: a prospective multivariate time-dependent analysis. J Hepatol 1993;18:185-95.

[5] Kaido T, Mori A, Ogura Y, Ogawa K, Hata K, Yoshizawa A, et al. Pre- and perioperative factors affecting infection after living donor liver transplantation. Nutrition 2012;28:1104-8.

[6] van Hoek B, de Rooij BJ, Verspaget HW. Risk factors for infection after liver transplantation. Best Pract Res Clin Gastroenterol 2012;26:61-72.

[7] Kim SI, Kim YJ, Jun YH, Wie SH, Kim YR, Choi JY, et al. Epidemiology and risk factors for bacteremia in 144 consecutive living-donor liver transplant recipients. Yonsei Med J 2009;50: $112-21$.

[8] Sun HY, Cacciarelli TV, Singh N. Identifying a targeted population at high risk for infections after liver transplantation in the MELD era. Clin Transplant 2011;25:420-5.

[9] Li C, Mi K, Wen TF, Yan LN, Li B, Wei YG, et al. Risk factors and outcomes of massive red blood cell transfusion following living donor liver transplantation. J Dig Dis 2012;13: $161-7$.

[10] Paugam-Burtz C, Kavafyan J, Merckx P, Dahmani S, Sommacale D, Ramsay M, et al. Postreperfusion syndrome during liver transplantation for cirrhosis: outcome and predictors. Liver Transpl 2009;15:522.

[11] Clemente WT, Romanelli RM, Faria LC, Lima SS, Jesus LA, Cortes JR, et al. Impact of model for end-stage liver disease in the occurrence of infectious events and survival in a cohort of liver transplant recipients. Transplant Proc 2013;45: 297-300.

[12] Patel G, Huprikar S. Infectious complications after orthotopic liver transplantation. Semin Respir Crit Care Med 2012;33: $111-24$.

[13] Pena JR, Barroso E, Martins A, Andrade JR. Sequential whole liver transplant resected as piggy-back from FAP patients (abstract) Liver Transpl 2002;8:C-24.

[14] Piselli P, Zanfi C, Corazza V, Ferretti S, Scuderi M, Arana $\mathrm{MG}$, et al. Incidence and timing of infections after liver transplant in Italy. Transplant Proc 2007;39:1950-2.

[15] Souza MV, Barth AL, Alvares-da-Silva MR, Machado AR. Infections after liver transplantation in adults: data from a university hospital in southern Brazil (1996-2000). Arq Gastroenterol 2007;44:128-32.

[16] Vera A, Contreras F, Guevara F. Incidence and risk factors for infections after liver transplant: single-center experience at the University Hospital Fundación Santa Fe de Bogotá, Colombia. Transpl Infect Dis 2011;13:608-15.

[17] Aberg F, Mäkisalo H, Höckerstedt K, Isoniemi H. Infectious complications more than 1 year after liver transplantation: a 3decade nationwide experience. Am J Transplant 2011;11:287-95. 
[18] Howard TK, Klintmalm GB, Cofer JB, Husberg BS, Goldstein RM, Gonwa TA. The influence of preservation injury on rejection in the hepatic transplant recipient. Transplantation 1990;49:103-7.

[19] Singh N, Wagener MM, Obman A, Cacciarelli TV, de Vera ME, Gayowski T. Bacteremias in liver transplant recipients: shift toward gram-negative bacteria as predominant pathogens. Liver Transpl 2004;10:844-9.

[20] Linares L, García-Goez JF, Cervera C, Almela M, Sanclemente G, Cofán F, et al. Early bacteremia after solid organ transplantation. Transplant Proc 2009;41:2262-4.
[21] Kawecki D, Chmura A, Pacholczyk M, Łagiewska B, Adadynski L, Wasiak D, et al. Etiological agents of bacteremia in the early period after liver transplantation. Transplant Proc 2007;39:2816-21.

[22] Hoyo I, Sanclemente G, Cervera C, Cofán F, Ricart MJ, Perez-Villa F, et al. Opportunistic pulmonary infections in solid organ transplant recipients. Transplant Proc 2012;44:2673-5.

[23] Van Thiel D, George M, Moore, et al. Fungal infections: their diagnosis and treatment in transplant recipients. Int J Hepatol 2012;2012:106923. 